\title{
Anisotropic flow decorrelation in heavy-ion collisions at RHIC-BES energies with 3D event-by-event viscous hydrodynamics
}

\author{
Jakub Cimerman, ${ }^{a, *}$ Iurii Karpenko, ${ }^{a}$ Boris Tomášik ${ }^{a, b}$ and Barbara Antonina \\ Trzeciak $^{a}$ \\ ${ }^{a}$ Faculty of Nuclear Sciences and Physical Engineering, Czech Technical University in Prague, \\ Břehová 7, 11519 Prague 1, Czech Republic \\ ${ }^{b}$ Univerzita Mateja Bela, Tajovského 40, 97401 Banská Bystrica, Slovakia \\ E-mail: jakub.cimerman@fjfi.cvut.cz
}

In the RHIC Beam Energy Scan program, gold nuclei are collided with different collision energies in the range from few to $62.4 \mathrm{GeV}$. The goals of the program are to explore the onset of QGP creation, locate the critical point of QCD and study dense baryon matter. We report on the first application of Monte Carlo Glauber (GLISSANDO2) and $\mathrm{T}_{\mathrm{R}}$ ENTo $p=0$ initial states extended to $3 \mathrm{D}$ for event-by-event viscous fluid dynamic (vHLLE) with hadronic cascade modelling of $\mathrm{Au}+\mathrm{Au}$ collisions at $\sqrt{s_{\mathrm{NN}}}=27$ and $62.4 \mathrm{GeV}$, which is the upper region of RHIC BES energies. The initial states are extended into both the longitudinal direction and for finite baryon density using simple ansätze. The full energy and baryon charge counting in the initial states is implemented. We show the reproduction of elliptic flow, at both collision energies and with both initial states. We compare it also to the results obtained with UrQMD initial state. Furthermore, we show the results for rapidity decorrelation of elliptic flow $r_{2}$ at $\sqrt{s_{\mathrm{NN}}}=27$ and $200 \mathrm{GeV}$ from the same setup of hydrodynamic calculations with the 3D Monte Carlo Glauber and UrQMD initial states. We discuss the features of the initial states responsible for the magnitude of the observed flow decorrelation.

\footnotetext{
*** The European Physical Society Conference on High Energy Physics (EPS-HEP2021), ***

*** 26-30 July $2021 * * *$

*** Online conference, jointly organized by Universität Hamburg and the research center DESY ***
}

\footnotetext{
${ }^{*}$ Speaker
} 


\section{Introduction}

For two decades, investigations of anisotropic flows taught us much about the quark-gluon plasma, but most of the studies focused on the flow in the transverse plane at midrapidity. However, studying event-by-event fluctuations along the longitudinal direction may help us understand the transport properties of quark-gluon plasma. At RHIC-BES energies, the decorrelation of the flow anisotropy along the longitudinal direction is just starting to be researched. So far, there are only preliminary results from STAR at $\sqrt{s_{\mathrm{NN}}}=27$ and $200 \mathrm{GeV}[1,2]$. This paper summarizes the first calculation of a kind at RHIC-BES energy in a hydrodynamic model [3].

\section{Model}

For the calculations a hybrid event-by-event viscous hydrodynamic model is used. It consists of four parts. It starts with three-dimensional initial state. Here, we worked with three different models: UrQMD [4], which uses PYTHIA6 to simulate inelastic nucleon-nucleon scatterings through string formation and subsequent string break-up, GLISSANDO2 [5], which is a Monte Carlo Glauber model, and $\mathrm{T}_{\mathrm{R}}$ ENTo [6], which introduces a generalized ansatz for the entropy density deposition from the participant nucleons. Since Glissando and $T_{R} E N T o$ only provide the initial state in the transverse plane, we extended them to longitudinal direction following [7]. Moreover, we fixed the total energy and baryon charge to those of participants and therefore there is one less parameter to tune. The hydrodynamic stage of evolution is modelled with a hydrodynamic code vHLLE [8], in which we included the shear viscosity. Next, a Monte Carlo hadron sampling is performed according to Cooper-Frye formula. The final step of the model is to simulate hadronic rescatterings and resonance decays of the sampled hadrons using UrQMD cascade. The model also includes a finite baryon and electric charge density at all stages and it is important, because we are doing simulations at relatively low energies. More detailed description of the model can be found in [9].

\section{Flow}

The classical definition of the anisotropic flow is through a Fourier series of $p_{T}$ distribution:

$$
v_{n}=\frac{\int \mathrm{d} \phi \cos \left(n\left(\phi-\Psi_{n}\right)\right) \frac{\mathrm{d}^{3} N}{p_{T} \mathrm{~d} p_{T} \mathrm{~d} y \mathrm{~d} \phi}}{\int \mathrm{d} \phi \frac{\mathrm{d}^{3} N}{p_{T} \mathrm{~d} p_{T} \mathrm{~d} y \mathrm{~d} \phi}} .
$$

There are several methods to calculate anisotropic flow from experimental or simulated data, from which the event plane (EP) method and the 2-particle cumulant method were used for this work. Figure 1 shows the centrality dependence of the $p_{T}$ integrated elliptic and triangular flow. From this figure we can see that $\mathrm{T}_{\mathrm{R}}$ ENTo IS produces the largest anisotropic flow, whereas GLISSANDO IS produces the lowest $v_{2}$ and $v_{3}$. In the non-central collisions at $27 \mathrm{GeV}$ as well as in all considered centrality classes at $62.4 \mathrm{GeV}$, calculations with $\mathrm{T}_{\mathrm{R}}$ ENTo IS have the best agreement with the experimental data for elliptic flow. However, $p_{T}$ dependent elliptic flow (Figure 2) shows that our calculations are in agreement with the data only for $p_{T}<1 \mathrm{GeV}$. At larger $p_{T}$, all of the initial states start to underpredict the data. 

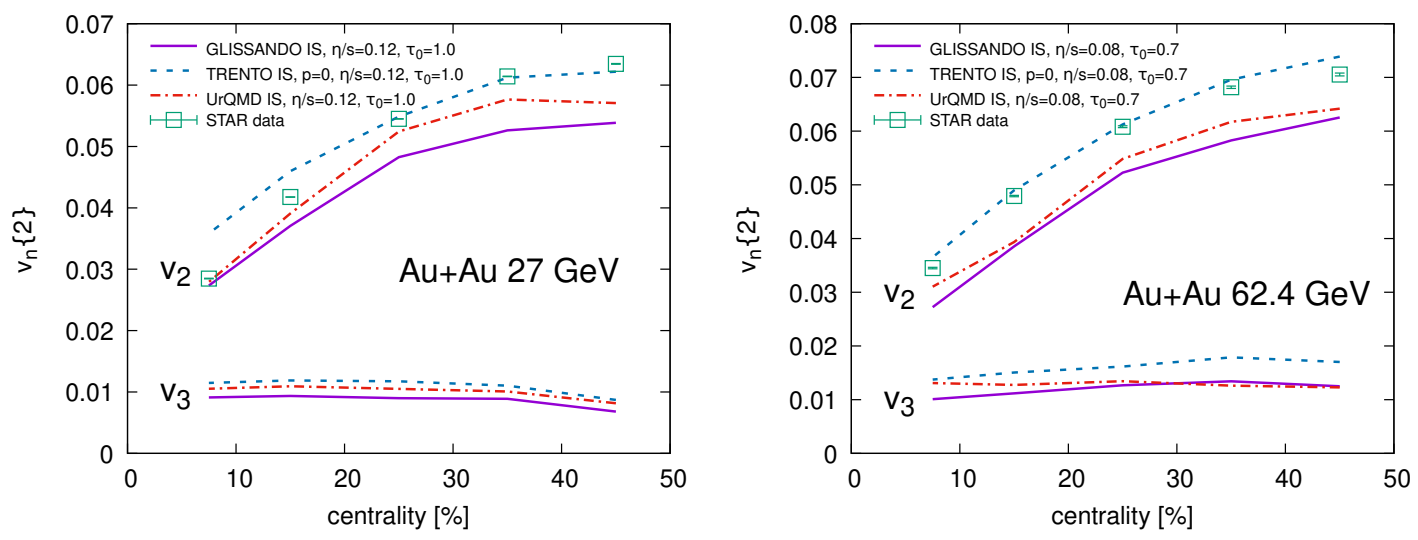

Figure 1: Elliptic and triangular flows of charged hadrons as functions of centrality for $\sqrt{s_{\mathrm{NN}}}=27$ (left) and 62.4 GeV (right) calculated using 2-particle cumulant method compared with STAR data [10, 11].
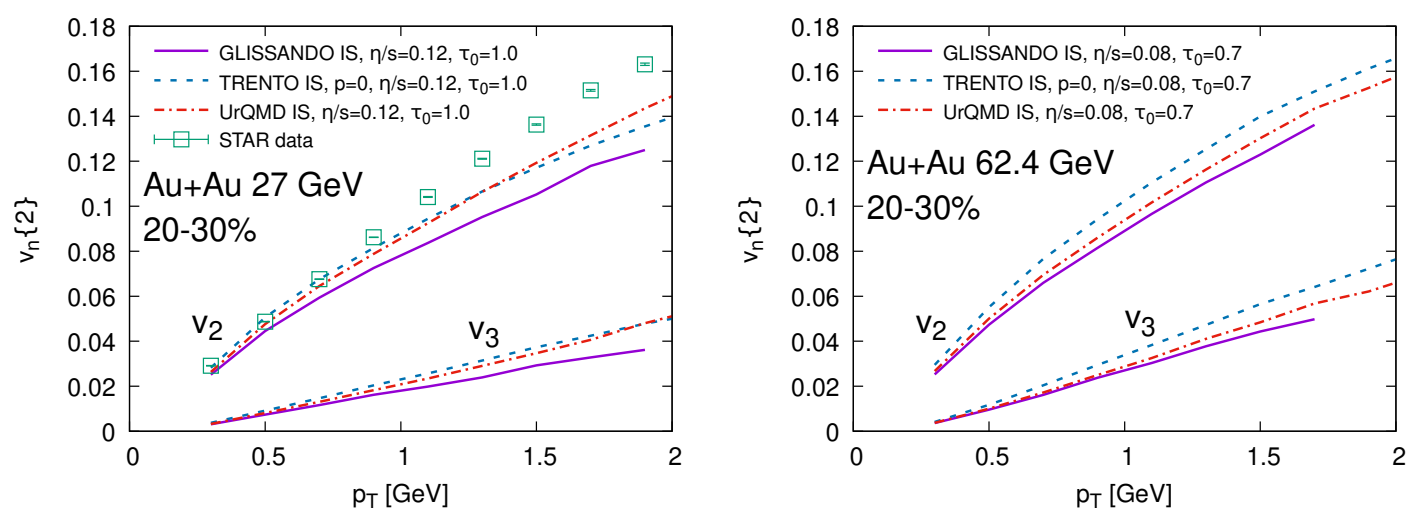

Figure 2: Elliptic and triangular flows of charged hadrons as functions of $p_{T}$ for $\sqrt{s_{\mathrm{NN}}}=27$ (left) and 62.4 $\mathrm{GeV}$ (right) calculated using 2-particle cumulant method compared with STAR data [10].

Figure 3 shows the elliptic flow as a function of pseudorapidity. At $27 \mathrm{GeV}$, GLISSANDO and UrQMD initial states reproduce overall order of magnitude of the elliptic flow, but underestimate its value at mid-rapidity, which is consistent with previous figures. At $200 \mathrm{GeV}$ the experimental data indicate a triangular pseudorapidity dependence that neither of the initial states can describe.

\section{Decorrelation}

The longitudinal fluctuations leads to decorrelation of anisotropic flows along the pseudorapidity direction. Using the definition of the flow vector $\mathbf{V}_{n}=v_{n} e^{i n \Psi_{n}}$, the longitudinal decorrelation can be expressed using the factorization ratio:

$$
r_{n}\left(\eta, \eta_{\mathrm{ref}}\right)=\frac{\left\langle\mathbf{V}_{n}(-\eta) \mathbf{V}_{n}^{*}\left(\eta_{\mathrm{ref}}\right)\right\rangle}{\left\langle\mathbf{V}_{n}(+\eta) \mathbf{V}_{n}^{*}\left(\eta_{\mathrm{ref}}\right)\right\rangle}=\frac{\left\langle v_{n}(-\eta) v_{n}\left(\eta_{\mathrm{ref}}\right) \cos n\left(\Psi_{n}(-\eta)-\Psi_{n}\left(\eta_{\mathrm{ref}}\right)\right)\right\rangle}{\left\langle v_{n}(+\eta) v_{n}\left(\eta_{\mathrm{ref}}\right) \cos n\left(\Psi_{n}(+\eta)-\Psi_{n}\left(\eta_{\mathrm{ref}}\right)\right)\right\rangle} .
$$

If the factorisation ratio is equal to 1 , it means that the flow vectors at $+\eta$ and $-\eta$ are fully correlated, and when it gets below 1, we got a decorrelation between flow vectors at $\pm \eta$. From Eq. (2) it 

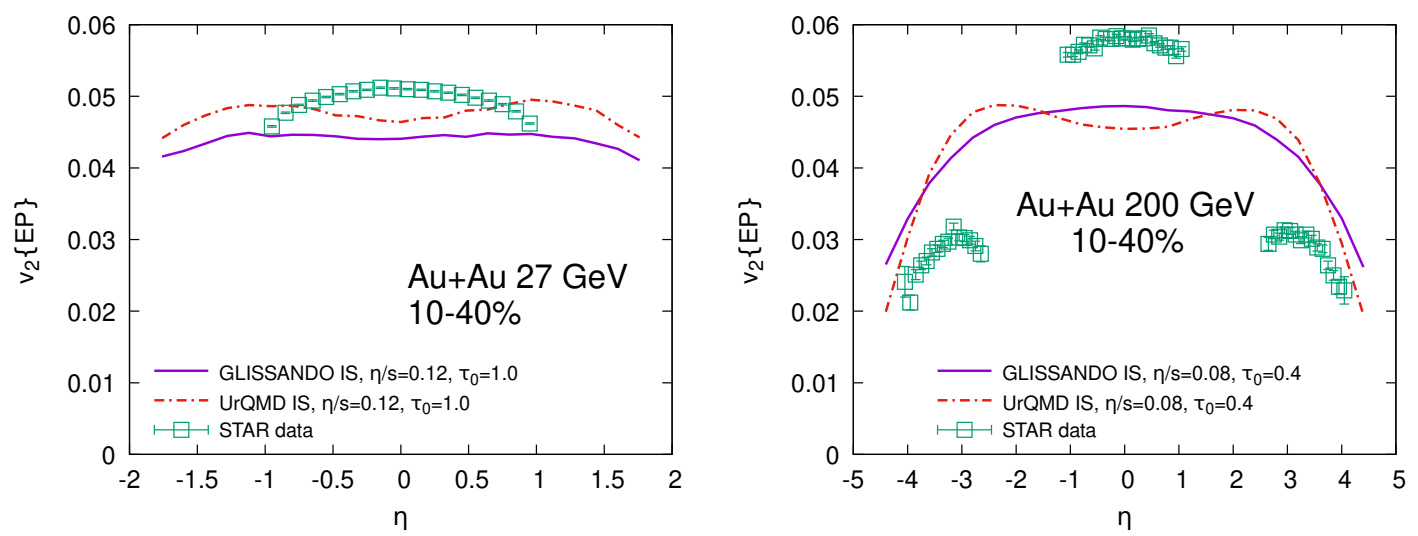

Figure 3: Elliptic flow of charged hadrons as a function of pseudorapidity for $\sqrt{s_{\mathrm{NN}}}=27$ (left) and $200 \mathrm{GeV}$ (right) calculated using EP method compared with STAR data [10, 12].

can be seen that the flow decorrelation may be caused by two separate effects: flow magnitude decorrelation and flow angle decorrelation. Thus we can define corresponding factorisation ratios:

$$
\begin{aligned}
r_{n}^{v}(\eta) & =\frac{\left\langle v_{n}(-\eta) v_{n}\left(\eta_{\mathrm{ref}}\right)\right\rangle}{\left\langle v_{n}(+\eta) v_{n}\left(\eta_{\mathrm{ref}}\right)\right\rangle}, \\
r_{n}^{\Psi}(\eta) & =\frac{\left\langle\cos n\left(\Psi_{n}(-\eta)-\Psi_{n}\left(\eta_{\mathrm{ref}}\right)\right)\right\rangle}{\left\langle\cos n\left(\Psi_{n}(+\eta)-\Psi_{n}\left(\eta_{\mathrm{ref}}\right)\right)\right\rangle} .
\end{aligned}
$$

Figure 4 shows the factorisation ratio as a function of pseudorapidity. We did not use the extended $\mathrm{T}_{\mathrm{R}}$ ENTo IS for these calculations, since it does not have implemented any tilt in the longitudinal direction and thus the factorisation ratio would be equal to 1 . At $27 \mathrm{GeV}$, the model with UrQMD initial state shows much stronger decorrelation then the one seen in the data. On the other hand, calculations with GLISSANDO initial state can describe the experimental data within uncertainties for both energies.
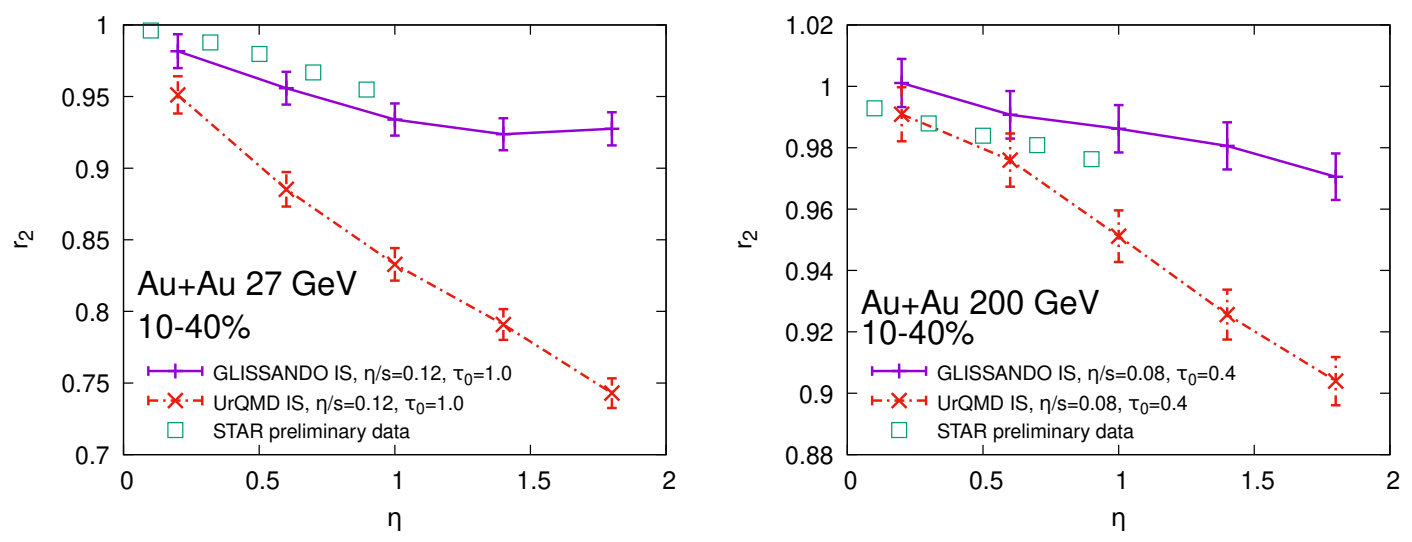

Figure 4: Factorization ratio $r_{2}$ of charged hadrons as a function of pseudorapidity for $10-40 \% \mathrm{Au}-\mathrm{Au}$ collisions at $\sqrt{s_{\mathrm{NN}}}=27$ (left) and $200 \mathrm{GeV}$ (right) compared with STAR preliminary data [1,2].

Figure 5 shows contributions of the flow angle and flow magnitude decorrelation separately for $27 \mathrm{GeV}$. It can be seen that for both models the flow angle decorrelation plays more important 
role than the flow magnitude decorrelation. The same hierarchy has been observed in calculations at LHC energies [13].

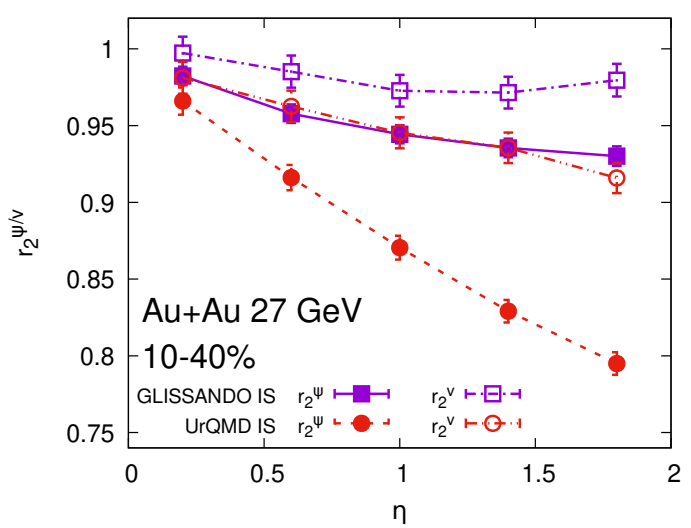

Figure 5: The flow magnitude $r_{2}^{v}$ and the flow angle decorrelation $r_{2}^{\Psi}$ of charged hadrons as a function of pseudorapidity for $10-40 \% \mathrm{Au}-\mathrm{Au}$ collisions at $\sqrt{s_{\mathrm{NN}}}=27 \mathrm{GeV}$.

It is well known that the anisotropic flow coefficients are strongly correlated with the initial state eccentricities. Therefore, to better understand the big difference between the initial state models, we can define the factorisation ratio of initial-state eccentricities:

$$
r_{n}^{\epsilon}\left(\eta_{s}\right)=\frac{\left\langle\epsilon_{n}\left(-\eta_{s}\right) \epsilon_{n}\left(\eta_{s, \text { ref }}\right) \cos \left[n\left(\Psi_{n}\left(-\eta_{s}\right)-\Psi_{n}\left(\eta_{s, \text { ref }}\right)\right)\right]\right\rangle}{\left\langle\epsilon_{n}\left(\eta_{s}\right) \epsilon_{n}\left(\eta_{s, \text { ref }}\right) \cos \left[n\left(\Psi_{n}\left(\eta_{s}\right)-\Psi_{n}\left(\eta_{s, \text { ref }}\right)\right)\right]\right\rangle}
$$

where

$$
\epsilon_{n} e^{i n \Psi_{n}}=\frac{\int e^{i n \phi} r^{n} \rho(\vec{r}) d \phi r d r}{\int r^{n} \rho(\vec{r}) d \phi r d r}
$$

This factorisation ratio as a function of space-time rapidity is shown in Figure 6. Comparison with the flow decorrelation (Figure 4) shows that the two factorisation ratios agree even quantitatively, which means that longitudinal decorrelation is created in the initial state.
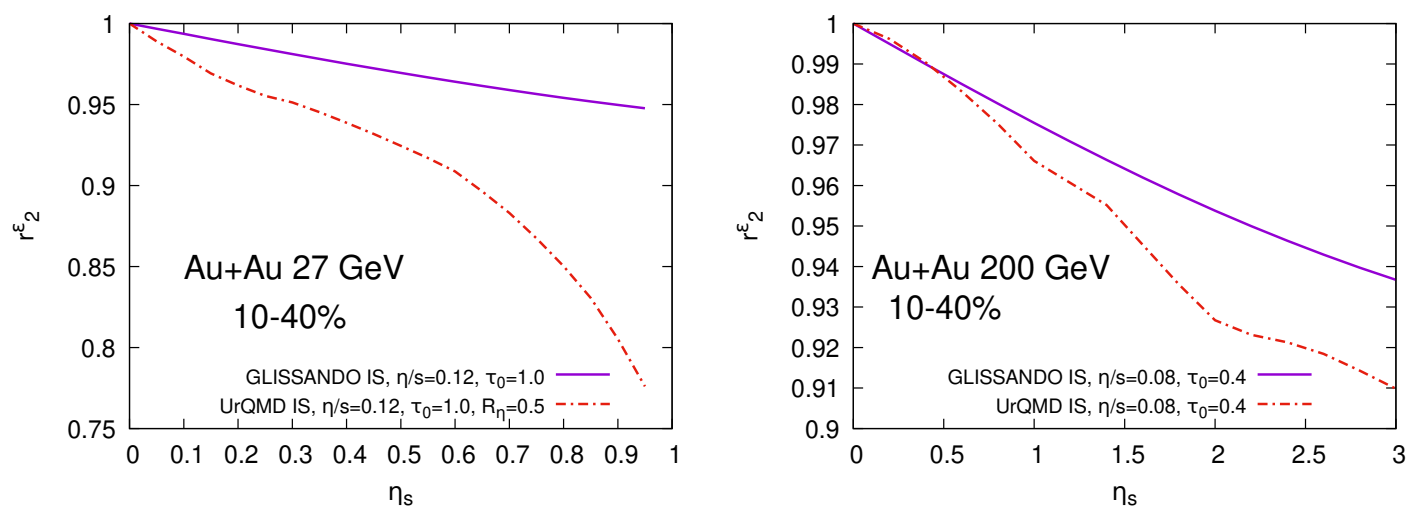

Figure 6: Longitudinal decorrelation of the initial state eccentricity $\epsilon_{2}$ as a function of space-time rapidity for $10-40 \% \mathrm{Au}-\mathrm{Au}$ collisions at $\sqrt{s_{\mathrm{NN}}}=27$ (left) and $200 \mathrm{GeV}$ (right). 


\section{Conclusions}

This paper presents the elliptic flow and the flow decorrelation in Au-Au collisions at RHICBES energies simulated with the help of 3D viscous hydrodynamic model with three different initial state alternatives, which is a first simulations with this kind of hydrodynamic model at these energies. The best description of the data at midrapidity has been provided by the model with $\mathrm{T}_{\mathrm{R}}$ ENTo initial state with parameter $p=0$. We found that the flow decorrelation is mainly caused by the decorrelation of the flow angle, which has been seen also at LHC energies. We also showed that the strong decorrelation of the model with UrQMD IS is caused by strong decorrelation of initial-state eccentricity.

\section{Acknowledgements}

JC, IK, and BT acknowledge support by the project Centre of Advanced Applied Sciences, No. CZ.02.1.01/0.0/0.0/16-019/0000778, co-financed by the European Union. JC and BAT acknowledge support from from The Czech Science Foundation, grant number: GJ20-16256Y. IK acknwowledges support by the Ministry of Education, Youth and Sports of the Czech Republic under grant "International Mobility of Researchers - MSCA IF IV at CTU in Prague" No. CZ.02.2.69/0.0/0.0/20_079/0017983. BT acknowledges support from VEGA 1/0348/18. Computational resources were supplied by the project "e-Infrastruktura CZ" (e-INFRA LM2018140) provided within the program Projects of Large Research, Development and Innovations Infrastructures.

\section{References}

[1] M. Nie [STAR collaboration], Nucl. Phys. A 982 (2019), 403-406

[2] M. Nie [STAR collaboration], Nucl. Phys. A 1005 (2021), 121783

[3] J. Cimerman et al., Phys. Rev. C 104, 014904 (2021)

[4] S. A. Bass et al., Prog. Part. Nucl. Phys. 41, 255-369 (1998)

[5] M. Rybczynski et al., Comput. Phys. Commun. 185, 1759-1772 (2014)

[6] J. S. Moreland, J. E. Bernhard and S. A. Bass, Phys. Rev. C 92, 011901 (2015)

[7] P. Bozek and W. Broniowski, Phys. Rev. C 85, 044910 (2012)

[8] I. Karpenko, P. Huovinen and M. Bleicher, Comput. Phys. Commun. 185, 3016-3027 (2014)

[9] J. Cimerman et al., Phys. Rev. C 103, 034902 (2021)

[10] L. Adamczyk et al. [STAR collaboration], Phys. Rev. C 86, 054908 (2012)

[11] L. Adamczyk et al. [STAR collaboration], Phys. Rev. C 98, 034918 (2018)

[12] B. I. Abelev et al. [STAR collaboration], Phys. Rev. C 77, 054901 (2008)

[13] P. Bozek and W. Broniowski, Phys. Rev. C 97, 034913 (2018) 